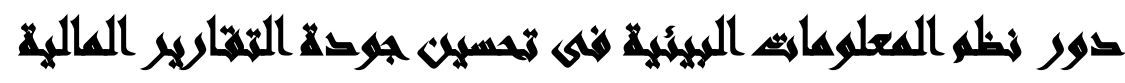

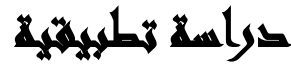

\section{[10]}

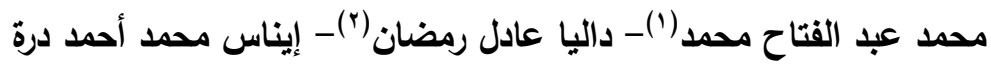

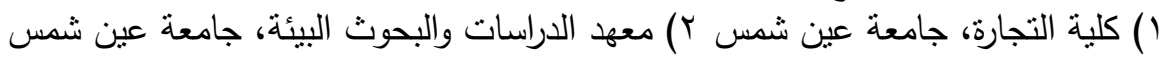

\section{المستخليه}

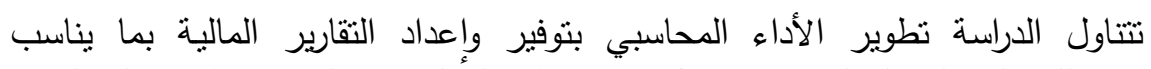

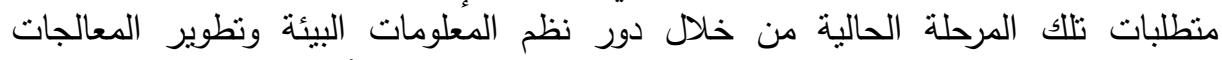

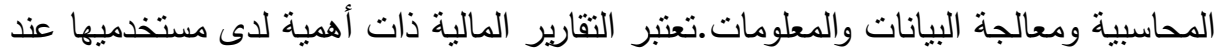

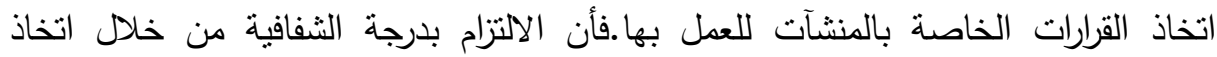

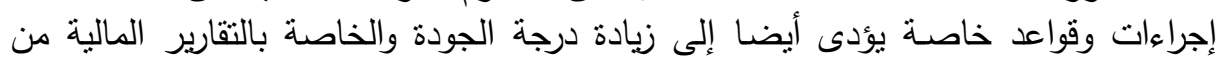
خلال نظم المعلومات البيأي. أهداف الدراسة: يتمنل الهُف العائين العام للبحث فى إبراز الدور الفعال لنظم المعلومات البيئية

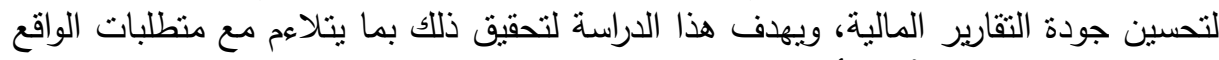

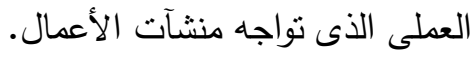

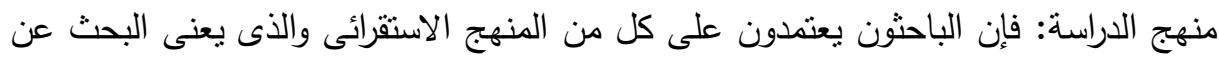

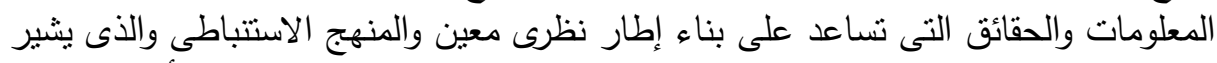

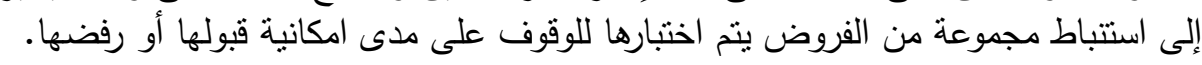

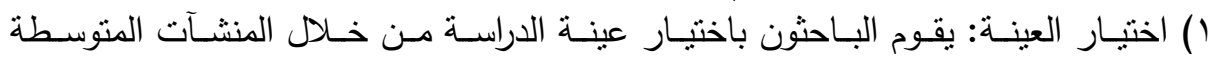
والصغيرة.

r) تجميع البيانات: حيث يتم تقديم استمارة استقصاه شاملة لكل الفئات التى تِعمل بالمنشآت التهات

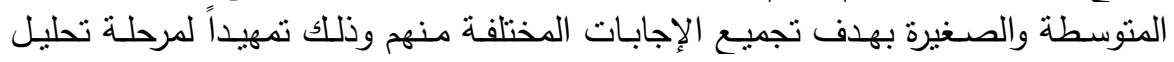
البيانات.

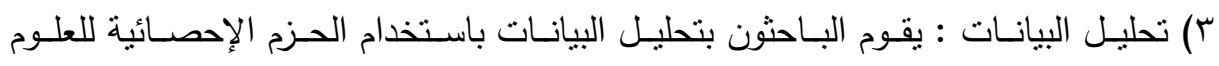

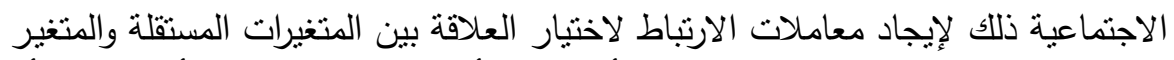

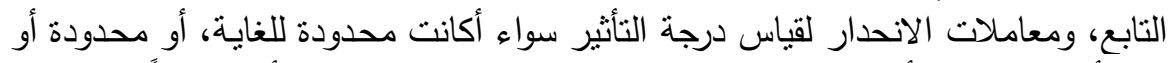
قوية أو قوية للغاية أما فى حالة كون المتغير لا يؤثر فتكون درجة التأثير صفراً.

$$
\text { المجلد السادس والثثلاثون، الجزء الأول، ديسمبر } 17 \text { ـ ا ب }
$$




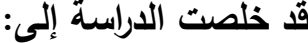

ا ـ وجود تأثثير ذو دلالة احصائية بين النظام الفعال للمعلومات البيئية وجودة التقارير المالية.

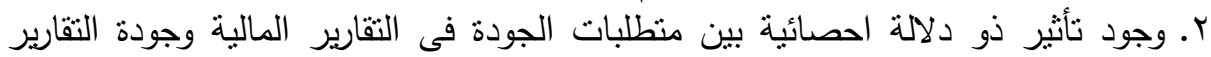

\section{rasadl}

فى ضوء الظروف الاقتصادية والاجتماعية النى تواجه المجتمع الذى نعيش فيه ، ومن

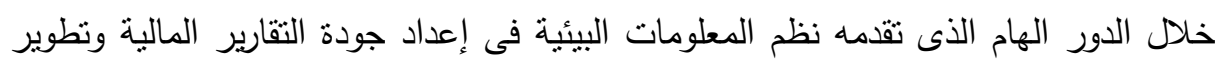

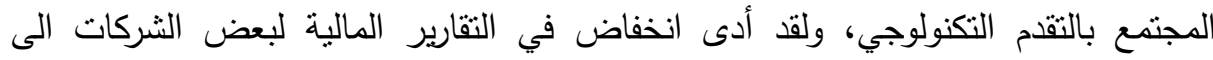
حالات إفلاس، مما دفع بالدول المتقدمة في السعي نحو إيجاد وسائل حديثة في مجال آليات تفعيل نظم الرقابة في الثركات المساهمة، وزيادة التقة فيما يتم إعداده ونشره من تقارير مالية فية وزيادة الثقة في تحسين جودة التقارير المالية للمنشآت.

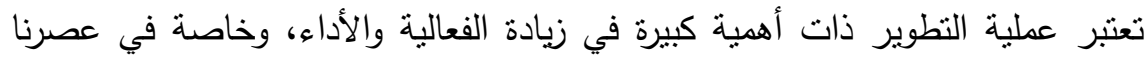
الحالي وذلك لاعنماد هذا العصر على البحث والدراسة والتحليل بغرض تطوير الجوانب والأبعاد التتظيمية ودل

ولقد وجد أنه من الضروري زيادة فعالية الإفصاح المحاسبي التى تصحبه الزيادة فى لئ إعداد التقارير المالية للعمل على كافة نظم المعلومات البيئية التى يمكن لمستخدمي تلك لكادئ

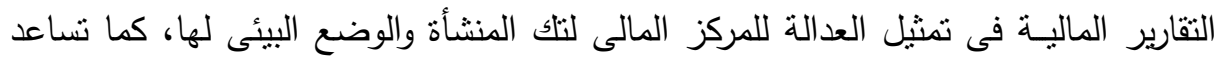
على تقييم الأداء البيئى مما يزيد فى سهولة اتخاذ القرارات الصحيحة والخاصة بها. أما عن مفهوم الجودة والثفافية ان تكون المعلومات والبيانات الخاصة بالتقارير المالية تعكس كل ما يجري ويدور داخل المنشأة الخاصة بالموضوع داخل إطار محاسبى للوصول إنول

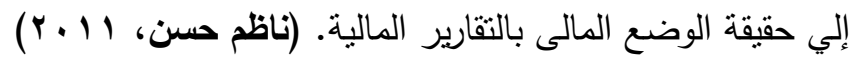
حيث يرى Janet Ranganat,1996 أن المجالات التى يجب أن تنتاولها هذه النقارير تختلف عن المجالات المتعارف عليها خاصة وأنها تعتمد على مجموعة من القياسات والبيانات غير التقليدية لم تكن مطلوبة فى إعداد التقارير السابقة ويؤكدون على أن التحدى على فئد 
الحقيقى أمام المنشآت هو إظهار الأفكار البيئية فى كل موقع يشغله أحد موظفيها وذلك

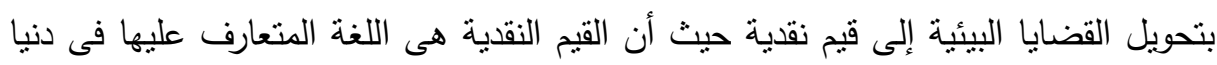
الأعمال وهى العنصر الحاكم عند اتخاذ القرار.

وقد أثنارت Marsden, Jack,1996 إلى أن أهم المخلفات على الإطلاق مخلفات

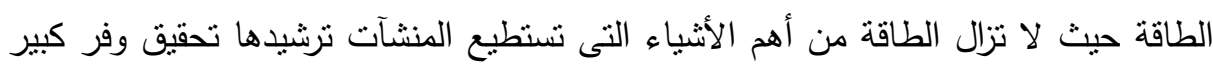

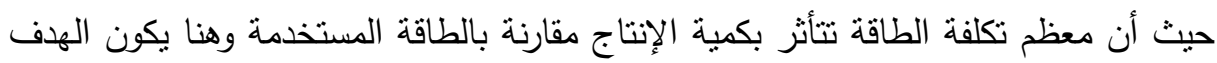

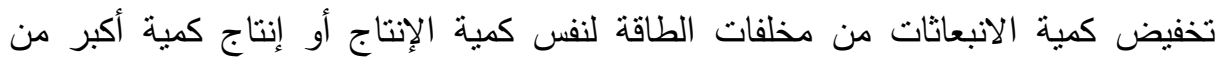

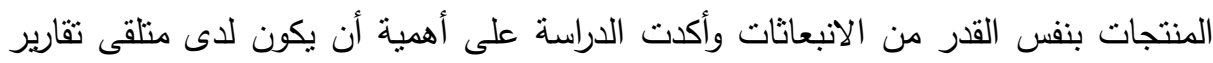

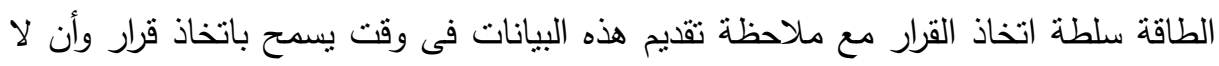
يكون ذلك بعد فوات الأوان.

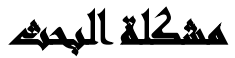

تمثل مشكلة البحث الاهتمام بتحسين التقارير المالية من خلال تطوير نظم المعلومات

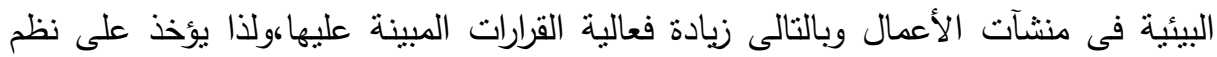
المعلومات فى مساهمتها بطرق غير مباشرة لما وصلت إليه البيئة من تدهور وذلك لعدم الالتزام أمام متخذى القرار بالوضوح والإفصاح والثفافية حتى يمكن تفادى التلوث البيائى، حيث أنه فى كثير من الأحيان تكون تكلفة إصلاح ما نم إتلافه أضعاف نكلفة التلوث نفسه. وقد أثنار Pennington, R إلى دور مراقب الحسابات فى فاعلية التقرير وان التقييم من خلال الخطوات التى يتبعها مراقب الحسابات واستخدام مراقب الحسابات مدخل المراجعة البينية مع إدارة المخاطر ولتحديد تلك المخاطر والإشارة إلى دور الإدارة فى تقييم التقرير دون

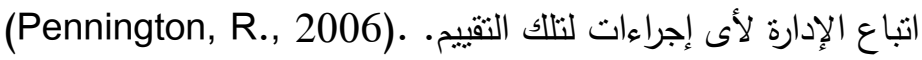

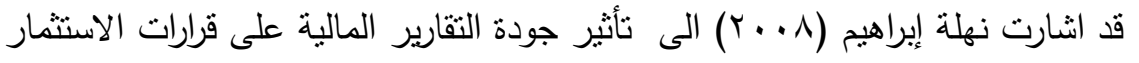
فى الأوراق المالية كما اهتمت بتعريف جديد لجودة التقارير المالية وهى عبارة عن استراتيجات وآليات تسهم فى الاهتمام بعملية إعداد التقارير من حيث الثكل والمضمون مما يجعلها قادرة على اتخاذ القرارات المناسبة. هدفت الى أن التقارير المالية الجيدة هى التى تتسم بالثفافية

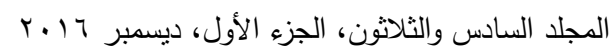


والإفصاح الجيد عن المعلومات التى تعكس حقيقة المراكز المالية والأرباح المحقة والمتوقعة للوحدات الاقتصادية بما يتفق مع احتياجاتها وأهدافها بالنسبة للمستثمرين. (نهلة إبراهيم، لهماته $\cdot(r \cdot \lambda$ قد اشار دراسة إلهام أبو غليطة أهمية التعرف على طبيعة العلاقة بين نظم المعلومات

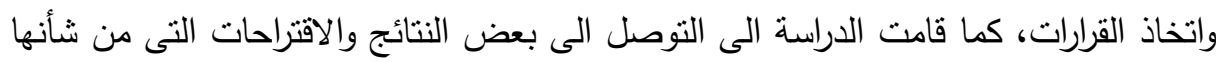

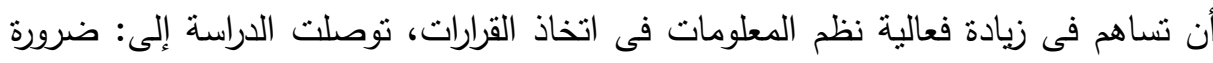

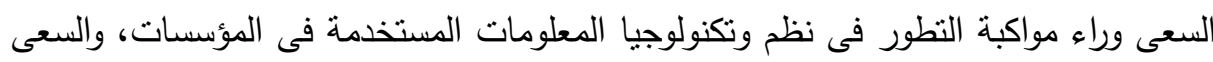

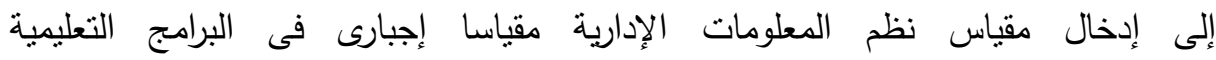

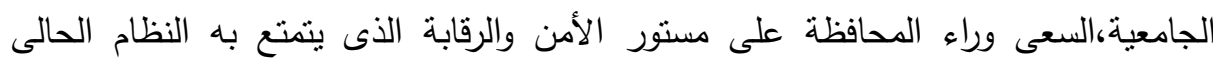

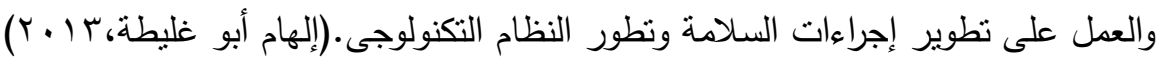

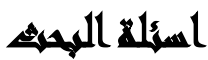

ما آلية تطبيق نظم المطومات البيئية لتحسين التقارير المالية؟ ويتفرع من خلال هذا السؤال الأسئلة التالية:

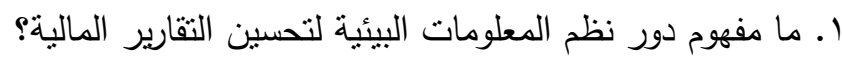

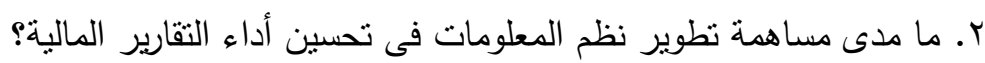

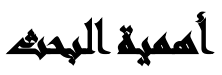

الأهمية العملية: تتشأ الأهمية العملية لهذا البحث بضرورة الاهتمام المتزايد من جانب مستخدمى التقارير المالية وذلك عن طريق الوضوح والإفصاح للأداء البيئى بمنشآت الأعمال. يرى الباحثون الأهمية البالغة للتعرف على المجالات والموضوعات التى يلزم عرضها بالتقارير الخاصة للأداء البيئى اللازمة فى الخياذ القالهة القرار لمستخدميها. 
من منطلق الأهمية التى يتتاولها البحث نطوير نظم المعلومات التى تساهم فى خفض

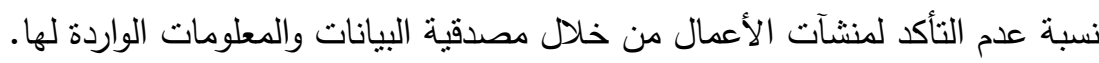
الأهمية العلمية: تأتى الأهية العلمية لهذه الدراسة لنطوير دور نظم المعلومات البيئية من أجل مواجهة المشكلات البيئية النى تواجه المجتمع الذى نعيش فيه.

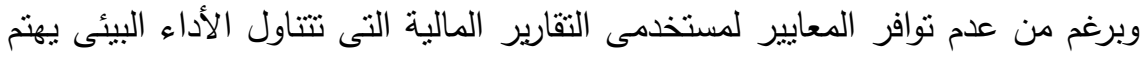
هذا البحث بالدراسة قدرة نظم المعلومات الحالية على إيجاد حلول تجد القدرة على توليد معلومات واضحة ذات مصدقية تعمل على خدمة إعداد تحسين الثقارير المالية التى تتتاول بيانات تتصل بقضايا البيئية . ولذا تقوم الأهمية العلمية على مساعدة القائمين على إعداد البرامج التى تتضمن الأنشطة والخدمات التى تساعد على إعادة نطوير نظم المعلومات البيئية من أجل الاستفادة فى عملية تحسين جودة التقارير المالية.

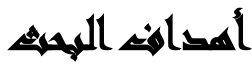

يتمثل الهدف العام للبحث فى إبراز الدور الفعال لنظم المعلومات البيئية لتحسين جودة التقارير المالية، ويهدف هذا البحث لتحقيق ذلك بما يتلاءم مع متطلبات الواقع العملى الذى لئى لئل تواجه منشآت الأعمال عن طريق الأهداف الفرعية التالية: 1 - دراسة مقومات تطبيق تاثير نظم المعلومات البيئية فى منشآت الأعمال. r- كيفية الاستفادة من جودة الإفصاح والثفافية لتحسين المعايير والخصائص النوعية للبيانات والمعلومات كمداخل للاستدلال على جودة التقارير المالية من خلال نظم

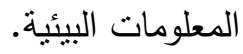
r- اقتراح الوسائل للقضاء على أوجه الخلاف بين ما يحتاجه متخذى القرار من بيانات

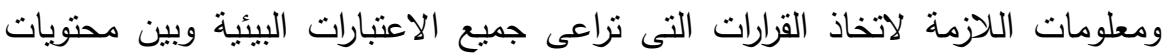


ع - إجراء دراسة نطبيقية للنأكد من تأثثر زيادة فعالية الإفصاح المحاسبى والبيئى فى تحسين جودة التقارير المالية ومدى تأثثر نظم المعلومات على تحديد كفاءة جودة التقارير المالية لاتخاذ القرارات الصحيحة لمستخدميها.

\section{هروض اللهمه}

في ضوء مشكلة وأهداف الدراسة يمكن صياغة الفروض التالية: • الفرض الرئيسى الأول: توجد علاقة ذات دلالة احصائية بين فعالية نظم المعلومات البيئية وجودة التقارير المالية. يتفرغ من الفرض الرئيسى الاول فرضين: الفرض الأول: توجد علاقة ذات دلالة إحصائية بين النظام الفعال للمعلومات البيئية وجودة لاونين التقارير المالية. الفرض الثانى: يوجد تأثير ذو دلالة إحصائية بين منطلبات الجودة فى التقارير المالية وجودة التقارير المالية.

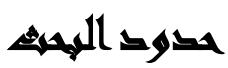

() تقتصر الدراسة على دراسة مدى امكانية دور نظام المعلومات البيئية فى تحسين جودة التقارير المالية. r) يتتاول الباحثون مجتمع المنشآت المنتسطة والصغيرة الصناعية وينم اختيار العينة من

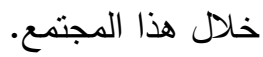




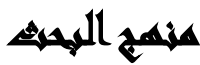

لتحقيق أهداف الدراسة فإن الباحثة سوف تعتمد على كل من المنهج الاستقرائى والذى يعنى البحث عن المعلومات والحقائق التى تساعد على بناء إطار نظرى معين والمنهج الاستتباطى والذى يشير إلى استتباط مجموعة من الفروض ينم اختبارها للوقوف على مدى لفى لفي امكانية قبولها أو رفضها. ويقوم المنهج الدراسة على جانبين أساسيين:

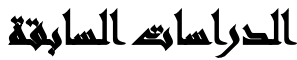

تعتبر الدراسات السابقة هي المرشد لكل باحث، وهي نقطة انطلاق بحثه عن طريقها

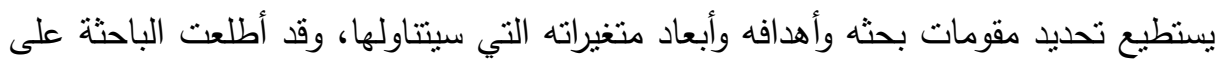
الدراسات السابقة العربية والأجنبية المتعلقة بعناصر الدراسة (نظم المعلومات البيئية وجودة ولهادية التقارير المالية) وفقاً للزهن من القديم للحديث.

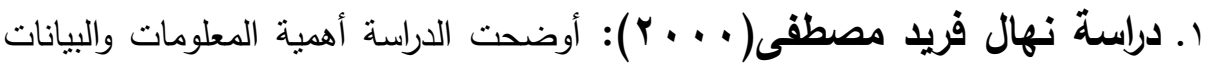
المالية التي تتضمنها التقارير المالية وبين المخاطر السوقية في قرارات الاستثمار. تحليل العلاقة بين البيانات المالية التى تتضمنها التقارير المالية وبين المخاطر السوقية فى قرارات الاستثمار فى الأوراق المالية. توصلت الدراسة إلى: أهية المعلومات المالية فى صنع واتخاذ قرارات الاستثمار والأوراق المالية فى حين أنه تتميز الأسواق المتقدمة بارتفاع فى درجة الوعى لدى المستثمرين.

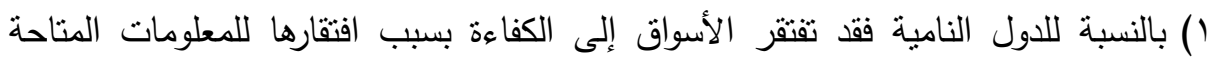
للمستثمرين على الرغم من أهمية البيانات والمعلومات المالية لتتبئوها بالمخاطر المالية

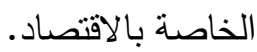

r) من أهم مقومات الأسواق الجيدة هو الإفصاح الجيد فى المعلومات حيث أنه لابد من توافر المعلومات الدقيقة عن المنشآت التى يجرى فيها التعامل على أوراقها من حيث الأبلى أرباحها وخططها المستقبلية وكفاءة إدارتها. يجب ارتفاع الوعى للمستثرين والمحللين بأهمية الاعتماد على المعلومات والبيانات الدقيقة.

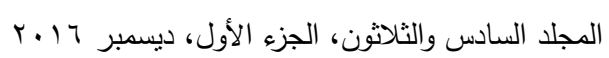




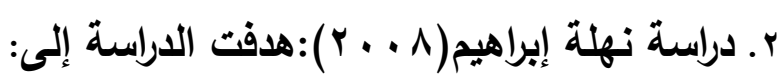

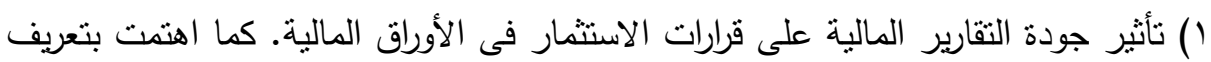
جديد لجودة التقارير المالية وهى عبارة عن استراتيجات وآليات نسهم فى الاهتمام بعملية إعداد التقارير من حيث الثكل والمضمون مما يجعلها قادرة على اتخاذ القرارات المناسبة.

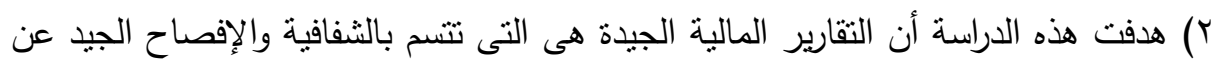

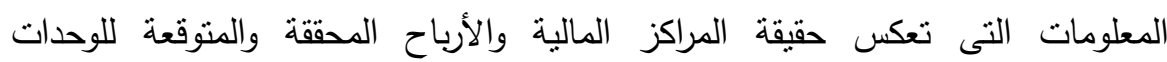
الاقتصادية بما يتفق مع احتباجاتها وأهدافها بالنسبة للمستثمرين.

$$
\text { توصلت الدراسة إلى: }
$$

() مراعاة تحقيق صحة المعلومات والإفصاح الجيد والوضوح والثنفافية بالثقارير المالية بما

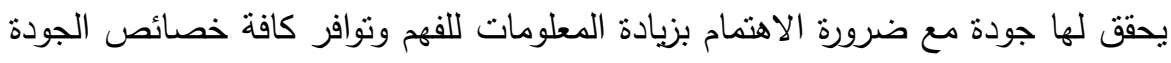
بالتقارير المالية والذى يعمل على تحقيق المواعمة بين حاجات المستخدمين الداخليين والخارجيين بما يحقق منفعة المعلومات المعلن عنها لكافة مستخدميها. r بؤكد على عدالة التقارير المالية والاهتمام والالتزام بنشر التقارير المالية لما لها من أهمات أهمية

$$
\text { فى اتخاذ القرارات نظراً لحداثة بياناتها ومعلوماتها. }
$$

r) كما أوصت الدراسة بمزيد من الأبحاث العلمية فى تقييم أداء جودة التقارير المالية.

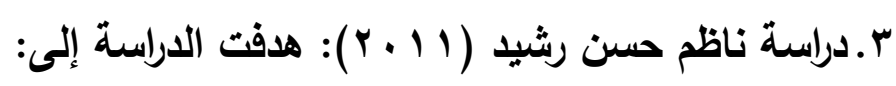

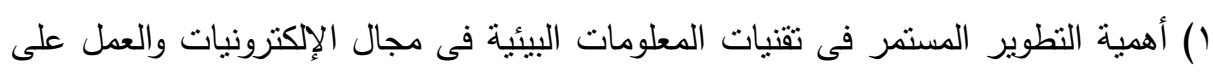

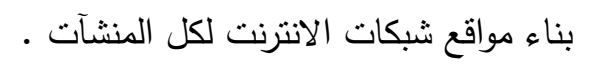

r) أهمية تقديم الاطار اللازم لدعم عملية النشر الالكترونى للبيانات المحاسبية البيئية. r) أهية تطوير النظام الالكتروني للإعداد الثقارير المالية وللنظام الدحاسبى.

$$
\text { وتوصلت الدراسة إلى: }
$$

( ) تحديث أسلوب شبكة نظم المعلومات والعمل بالتقنيات الجديدة بها، وكيفية استخدام النشر الالكترونى من اجل تطوير التقارير المالية لاتخاذ القرارات اللازمة. 
r) تحديد دور مراقب الحسابات فى الثقة بالبيانات المحاسبية للحصول على البيانات المالية

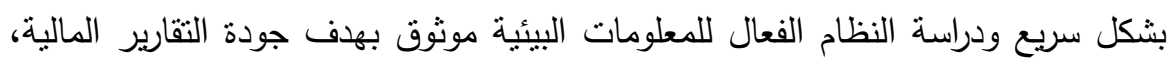

وتخفيض التكلفة والجهد فى جمع تحليل البيانات عن طريق نظم المعلومات البيئية .

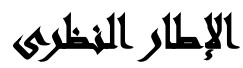

الخلفية النظرية للبحث:

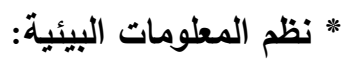

ا. رأي ( loudan 1999 أن نظام المعلومات يثير إلى دعم عطليات صنع القرار وتحقيق

r. قد أكد أحمد مصطفى أحمد بوابة، أن الاهتمام بالمعلومات هي أهم المقومات الأساسية في الحياة فتعتبر المعلومات هي شريان الحياة لانها نساعد متخذب القرار في تحقيق النتائج.

r. أما (sahwartr 1999) فإنه أكد أن نظم المعلومات بأنه نظام الأفراد والمعدات والإجراءات والمستتدات ووسائل الاتصال الذي يجمع البيانات ويقوم بعمليات تشكيل وتمرين واسترجاع وعرض البيانات لاستخدامها في التخطيط والموازنات الخططية.

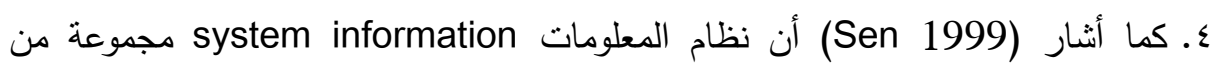
الإجراءات التي تتفاعل من أجل معالجة البيانات وتحويلها إلى معلومات في استخدام صنع القرارات.

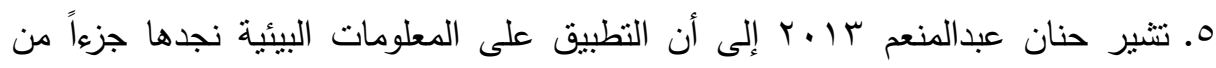

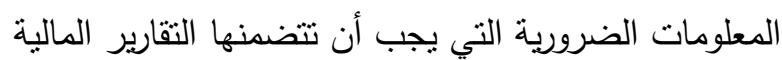

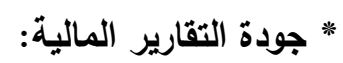
ا ـ أما ( Nashwa,G.2003) أن جودة التقارير المالية هي توافر معايير محاسبية والتي يتم على أساسها إعداد التقارير المالية بما يمكن للمستخدمين ومتخذ القرار من اتخاذ القرارات الرشيدة.

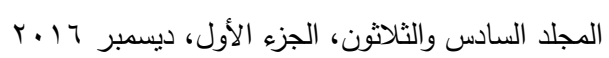


r. كما يرى ( carey \& parker 2003 أنه يتوافق مدى الثفافية على كمية جودة المعلومات المنشودة المتاحة لمستخدميها

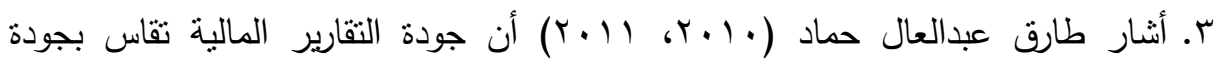
المعلومات المحاسبية والتي يتم الافصاح عنها في تلك التقارير حيث تكون مفيدة للمستخدمين في اتخاذ القرارات.

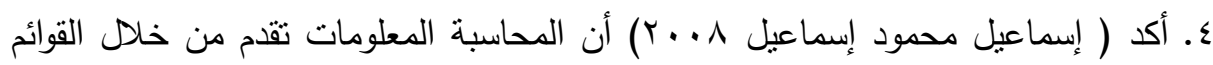
المالية التي تعتبر مخرجات النظام المحاسبي وبالتالي فالقوائم المالية تعتبر رسالة المنشأة

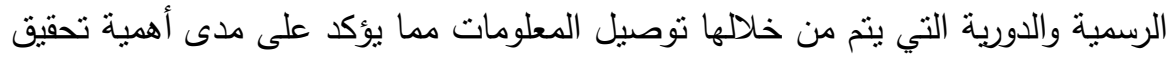
الجودة في التقارير المالية لأنها أداء داعمة في اتخاذ القرارات الرشيدة لجميع مستخدميها.

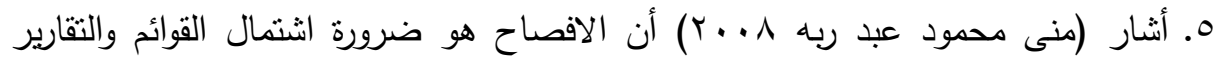

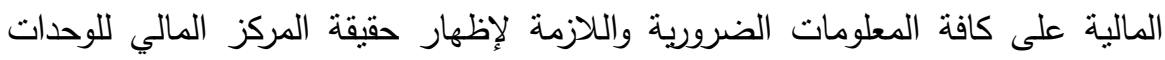
الاقتصادية وتقديم تلك المعلومات لمن يحتاج إليها بهدف المساعدة في اتخاذ القرارات

\section{المبحث الثاني: الاراسة الميدانية ونفية}

\section{مجتمع وعينة الدراسة:}

مجتمع الاراسة: يتمنل مجتمع الدراسة في المنثآت المتوسطة والصغيرة الصناعية. فيما بخص عينة الدراسة: المحاسبين والعاملين فى الإدارة المالية والمنشآت الصناعية، وتم اختيار هذه الفئة لاستطلاع أرائهم حول مدى فاعلية دور المعلومات البيئية فى تحسين جودة البئ التقارير المالية، حيث تعتبر هذه الفئة من الأطراف المعنية بهذا الشأن.

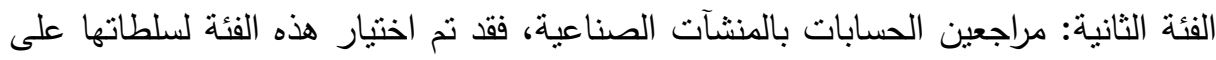
الرقابة لاستطلاع آرائهم حول فاعلية دور نظم المعلومات البيئية فى تحسين جودة التقارير

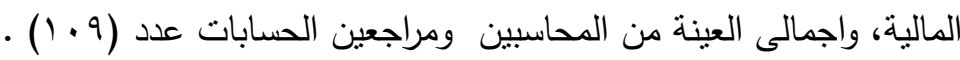

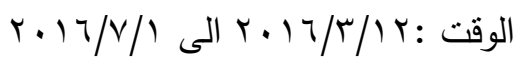




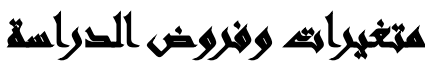

من خلال الإطلاع على الأبحاث وجد الباحثون أن الدراسات لم تتتاول بشكل كاف دور نظم المعلومات البيئية فى تحسين جودة النقارير المالية وبناءاً على ذلك قام الباحثون بتحديد المتغيرات المستقلة والمتغير التابع وفروض الدراسة فى هذه الدراسة كما يلى: (أ) المتغيرات المستقلة والمتغير التابع:

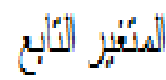

Dependent variable

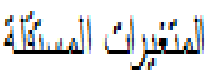

Independent variables

النظام الفعال للمعلومات البية:

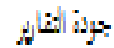

مابة

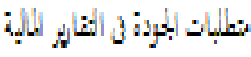

النموذج المقترح لنظم الإدارة البيئية فى تحسين جودة التقارير المالية

(ب) فروض الاراسة: تقوم الدراسة على اختبار الفروض الآتية:

الفرض الرئيسى الأول: توجد علاقة ذات دلالة احصائية بين فعالية نظم المعلومات البيئية وجودة الثقارير المالية، ويتفرغ من الفرض الرئيسى الاول فرضين فرعيين: 
الفرض الأول: نوجد علاقة ذات دلالة إحصائية بين النظام الفعال للمعلومات البيئية وجودة التقارير المالية.

الفرض الثانى: يوجد تأثثر ذو دلالة إحصائية بين منطلبات الجودة فى الثقارير المالية وجودة التقارير المالية. نتائج الدراسة: اثبات صحة الفرض الاول الرئيسى الذى ينص على نوجد علاقة ذات دلالة

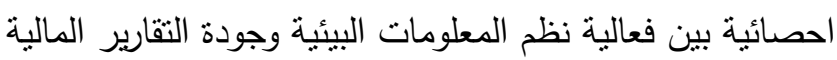

جدول رقم(1): علاقات الارتباط بين فعالية نظم المعلومات البيئية وجودة النقارير المالية

\begin{tabular}{|c|c|c|}
\hline جودة التقارير المالية & معامل الارتباط & المتغيرات \\
\hline$(* *) \cdot$, oV & معامل الازتباط الخطي & \multirow{2}{*}{ النظام الفعال للمعلومات البيئية } \\
\hline$\cdot, \cdots 1$ & مستوى المعنوية & \\
\hline$(* *) \cdot, \vee \vee T$ & معامل الارتباط الخطح & \multirow{2}{*}{ متطلبات الجودة فى النقارير المالية } \\
\hline$\cdot, \ldots 1$ & مستوى المعنوية & \\
\hline
\end{tabular}

** Correlation is significant at the 0.01 level (2 ،tailed).

يوضح الجدول السابق وجود علاقة ارتباط ذات دلالة معنوية بين فعالية نظم المعلومات

البيئية وجودة التقارير المالية

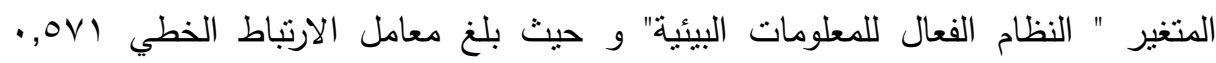
بمستوى معنوية | (., ب...

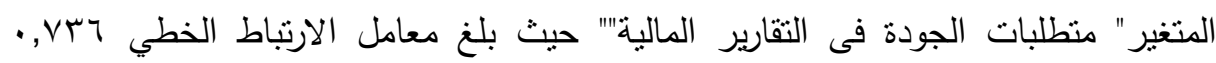
بمستوى معنوية ال .,.,..

الفرض الثانى الرئيسى: يوجد تأثثر ذو دلالة احصائية بين فعالية نظم المعلومات البيئية وجودة التقارير المالية 
جدول رقم(ץ): نتائج الارتباط والاتحدار المتعدد بين متطلبات بين فعالية نظم المعلومات البيئية وجودة الثقارير المالية

\begin{tabular}{|c|c|c|c|c|c|}
\hline المعنوية مستوى & المحسوية & الانحدار معامل & المعياري & 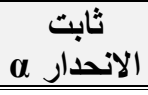 & \\
\hline., .10 & $r, \leqslant 70$ & & 1,709 & $\varepsilon, \wedge 9$ & الثابت \\
\hline$\left({ }^{*}\right),, \ldots 1$ & סחז, & •, Y97 & •, & ع & للمعلومات البيئية \\
\hline$\left({ }^{*}\right), \ldots 1$ & $\wedge, \wedge$ & $\cdot, 7$ & $\cdot, \cdot Y \Lambda$ & $\cdot, r \leq 0$ & منطلبات الجودة فىى التقارير المالية \\
\hline
\end{tabular}

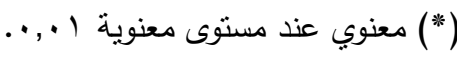

يتضح من الجدول ما يلي: أظهرت نتائج الارتباط المتعدد وجود علاقة موجبة ذات دلالة

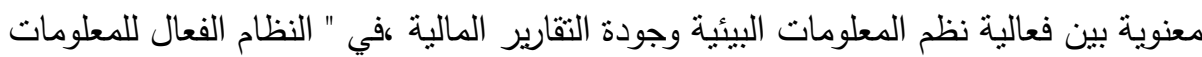
البيئية، منطلبات الجودة فى التقارير المالية" كالاتى:

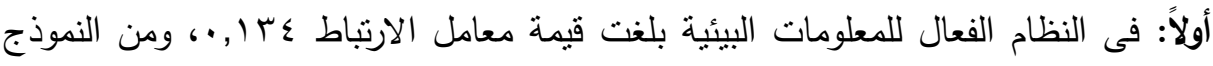

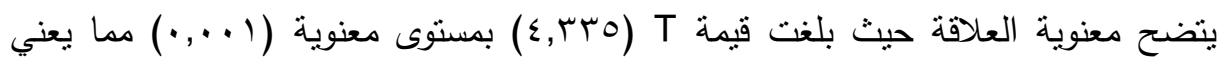
معنويتها عند مسنوى معنوية (1 (., ))، أي وجود تأثير ذي دلالة معنوية لوجود النظام الفعال

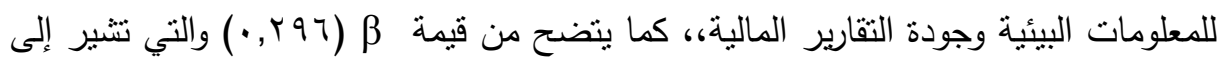

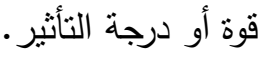

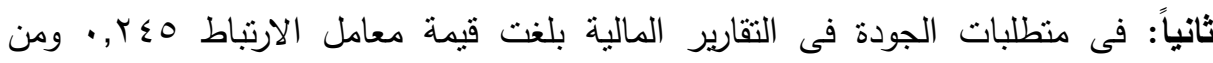

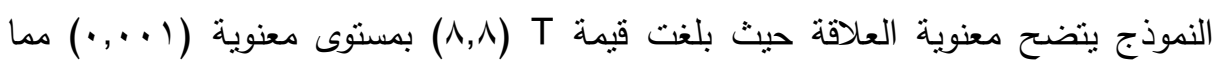
يعني معنويتها عند مستوى معنوية (1 ., •)، أي وجود تأثنير ذي دلالة معنوية لوجود منطلبات

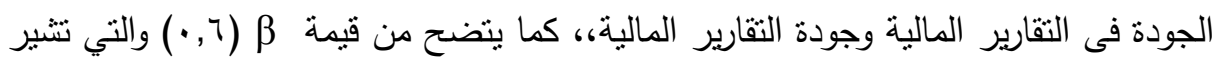

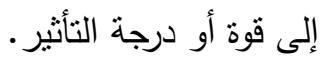
ويتفرع من الفروض الرئيسيى الثانى فرضين: الفرض الفرعى الأول: يوجد تأثثر ذو دلالة احصائية بين النظام الفعال للمعلومات البيئية وجودة التقارير المالية 
جدول رقم(ץ): نتائج الارتباط والانحدار بين النظام الفعال للمعلومات البيئية وجودة التقارير المالية

\begin{tabular}{|c|c|c|c|c|c|c|c|}
\hline \multicolumn{8}{|c|}{ 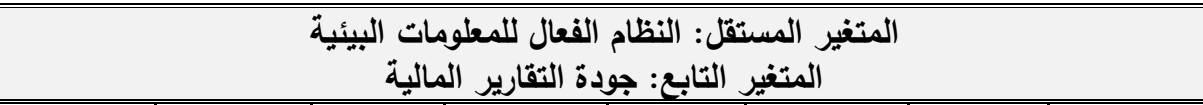 } \\
\hline 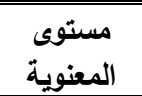 & قالمحسوية & المعامل & المعاملات & \multirow{2}{*}{ 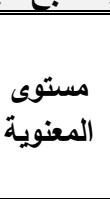 } & \multirow{2}{*}{ F } & \multirow{2}{*}{$\begin{array}{l}\text { معامل } \\
\text { R2 }\end{array}$} & \multirow{2}{*}{$\begin{array}{c}\text { الارتباط } \\
\text { Rعل }\end{array}$} \\
\hline$(*), \ldots 1$ & $r, 0 . r$ & $v, \leqslant 11$ & 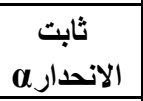 & & & & \\
\hline$(*), \ldots 1$ & $V, r \cdot r$ & $\cdot, Y \otimes \wedge$ & 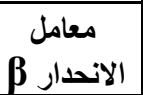 & $\cdot, \ldots 1$ & $01, \wedge \wedge 1$ & •, rrv & $(*) \cdot, \circ \vee 1$ \\
\hline
\end{tabular}

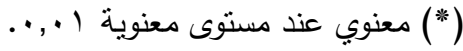

يتضح من الجدول ما يلي: أظهرت نتائج الارتباط البسيط وجود علاقة موجبة ذات دلالة

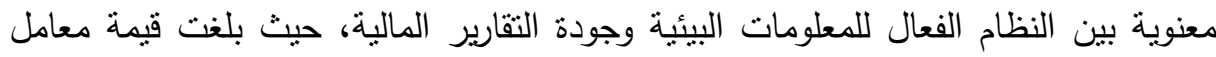
الارتباط اOV/, بمستوى معنوية I .,., مما يعني معنوية العلاقة عند مستوى معنوية $\therefore, \cdot 1$

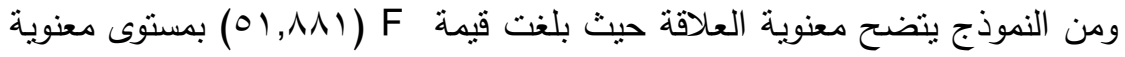

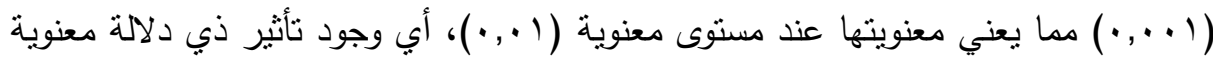

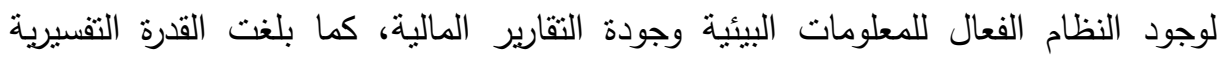
للنموذج (r\%\%) وذللك من خلا قيمة R²، أي أن نسبة (rr\%) من التغيرات التي تحدث

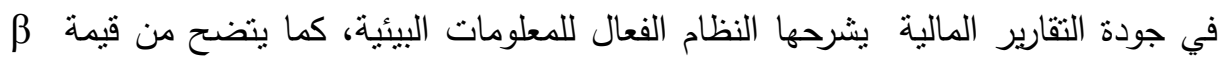

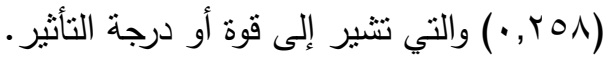
الفرض الفرعى الثانى: يوجد ناثير ذو دلالة احصائية بين منطلبات الجودة فى التقارير المالية وجودة التقارير المالية 
جدول رقم(؛): نتائج الارتباط والانحدار بين منطلبات الجودة فى التقارير المالية وجودة التقارير المالية

\begin{tabular}{|c|c|c|c|c|c|c|c|}
\hline \multicolumn{8}{|c|}{ 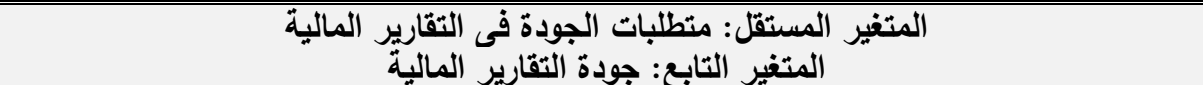 } \\
\hline 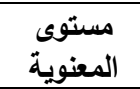 & المحسوية & قالمعامل & المعاملات & \multirow{2}{*}{ المعنوية } & \multirow{2}{*}{ المحسوية } & \multirow{2}{*}{$\begin{array}{l}\text { التحديد } \\
\text { R2 }\end{array}$} & \multirow{2}{*}{$\begin{array}{c}\text { الارتباط } \\
\text { R }\end{array}$} \\
\hline$(*) \cdot, \ldots$ & 1,111 & Q,OYT & الانحدارة & & & & \\
\hline$(*) \cdot, \ldots$ & $11, r o$. & $\cdot, \mu$ & الانحدار B معل & $\cdot, \ldots 1$ & אO, & $\cdot, 0 \leqslant r$ & $\left({ }^{*}\right) \cdot, V \vee T$ \\
\hline
\end{tabular}

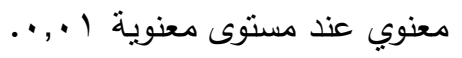

يتضح من الجدول ما يلي: أظهرت نتائج الارتباط البسيط وجود علاقة موجبة ذات دلالة معنوية بين متطلبات الجودة فى التقارير المالية وجودة التقارير المالية، حيث بلغت قيمة معامل الارتباط بrV, • بمستوى معنوية I .,., مما بعني معنوية العلاقة عند مستوى معنوية $\cdot \cdot, \cdot 1$

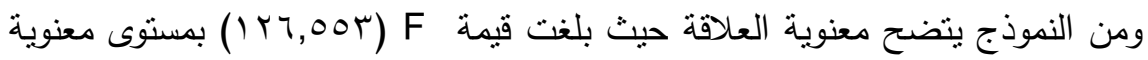

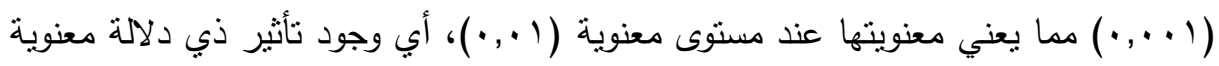
لوجود متطلبات الجودة فى التقارير المالية وجودة التقارير المالية، كما بلغت القدرة التفسيرية

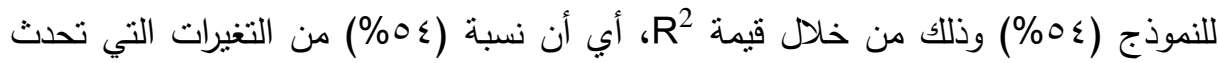

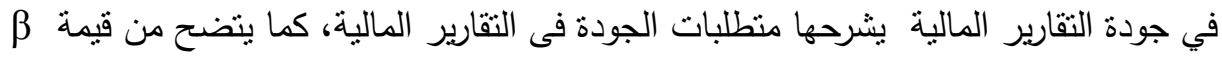

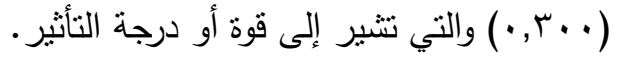

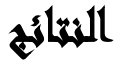

ا ـ أن نظم المعلومات البيئية في المنشآت الصناعية تؤثر تأثثراً إيجابياً في اتخاذ القرارات.كما

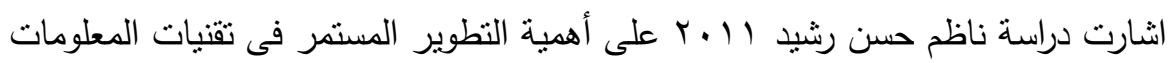

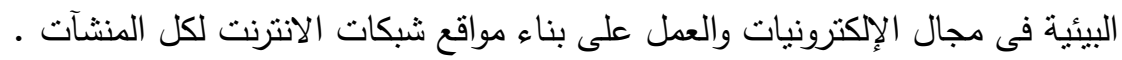

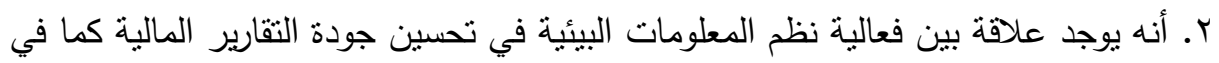
دراسة نهلة ابراهيم ^ . . r حيث ان التقارير المالية الجيدة التى تتسم بالثفافية والإفصاح

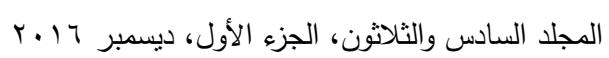


الجيد عن المعلومات تعكس حقيقة المراكز المالية والأرباح المحققة والمنوقعة للوحدات الاقتصادية بما يتفق مع احتياجاتها وأهدافها بالنسبة للمستثمرين.

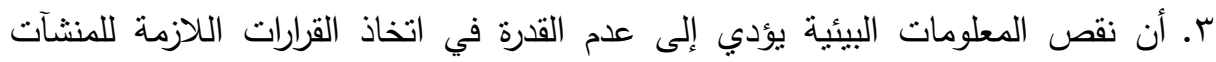

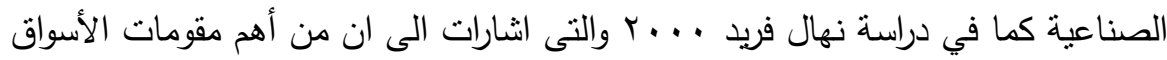

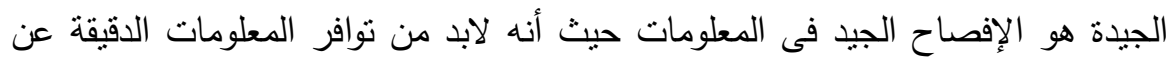

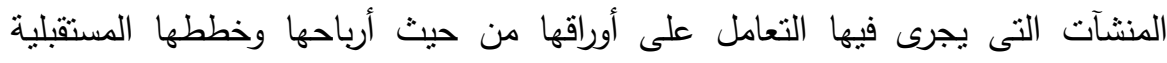
وكفاءة إدارتها. ع. التحقق من عدم وجود أخطاء أو تلاعب في المعلومات المحاسبية المستخدمة للتقارير

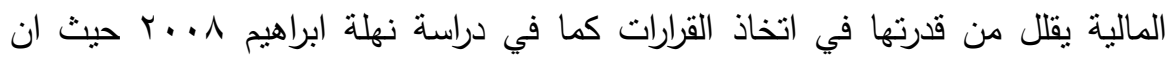
التقارير المالية الجيدة التى تتسم بالثفافية والإفصاح الجيد عن المعلومات تعكس حقيقة

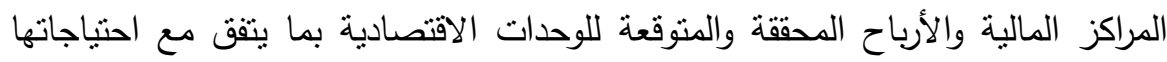
وأهدافها بالنسبة للمستتمرين.

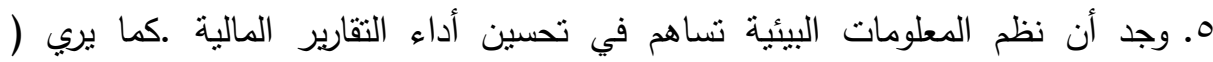
(loudan 1999 أن نظام المعلومات يثير إلى دعم عمليات صنع القرار وتحقيق

7. أنه يمكن قياس تحسين جودة التقارير المالية عن طريق النطوير التكنولوجي المستمر لنظم

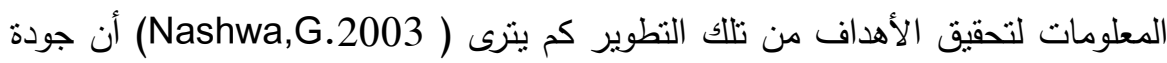
التقارير المالية هي توافر معايير محاسبية والتي يتم على أساسها إعداد التقارير المالية بما يمكن للمستخدمين ومتخذ القرار من اتخاذ القرارات الرشيدة. نتائج أختيار صحة الفروض الرئيسية: ثم قبول الفرض الرئيسي الأول للاراسة الذي أثبت أنه

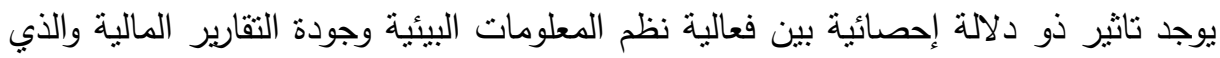
تفرع منه فرضين فرعين: أولاً: ثم قبول الفرض الأول الفرعي: يوجد تأثير ذو دلالة إحصائية بين النظام الفعال للمعلومات البيئية وجودة التقارير المالية. 
ثانياً: ثم قبول الفرض الثاني الفرعي حيث أنه نأثير ذو دلالة إحصائية بين متطلبات الجودة في التقارير المالية وجودة النقارير المالية. حيث أكلات النتائج على أنه:

- وجود علاقة بين فعالية نظم المعلومات البيئية وجودة التقارير المالية - وجود تأثثر بين نظم المعلومات البيئية وجودة التقارير المالية

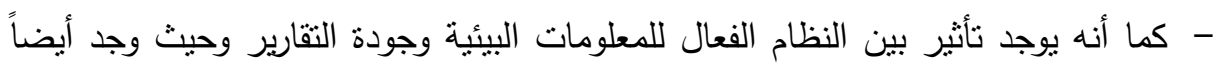
تأثثر بين منطلبات الجودة في التقارير وجودة التقارير المالية.

\section{التحوصياهت}

ظهرت النتائج بأن نظم المعلومات البيئية هو شريان الحياة الحقيقي لتحسين التقارير المالية لذا نوصي الباحثة باتباع التالي:

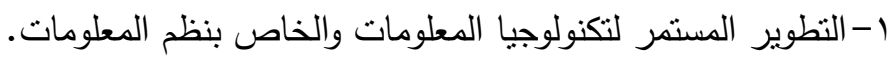

ץ-المصداقية والوضوح لتحقيق الجودة في المعلومات والبيانات التي تؤثز على اتخاذ القرارات.

r- المزيد من الإفصاح والثفافية للتقارير المالية لتأثيرها في اتخاذ القرارات. ع-يقترح الباحثون العمل على المقارنة للتقارير المالية لسنوات متعددة لزيادة كفاءة البيانات والمعلومات وملاءمتها لخدمة توقعات المنشآت الاقتصادية مستقبلياً.

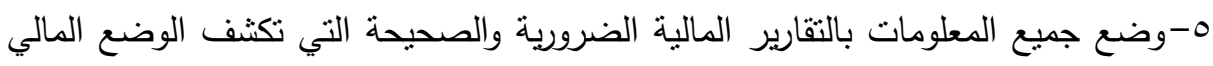
لتلك التقارير المستقبلية والحالية للمنشآت الاقتصادية. צ-يقترح الباحثون سرعة تحديث البيانات الدائم للمساهمة في اتخاذ القرارات اللازمة للمنشآت الاقتصادية.

V-يقترح الباحثون الاهنمام بالدقة في المعلومات المحاسبية الواردة في التقارير المالية

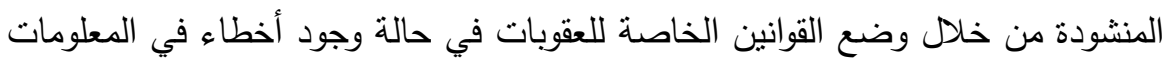
( وضع معلومات مغلوطة) التي تعمل على التأثثر في اتخاذ القرارات.

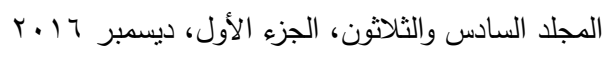


ᄉ-يقترح الباحثون التشجيع على الأبحاث والدراسات التي تهدف إلى الوعي المستمر للتوصل

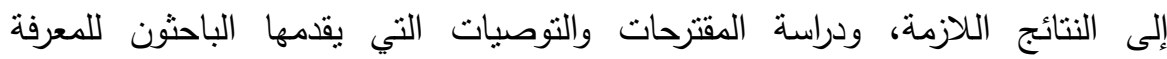

\section{exlall}

أسامة عبد اللطيف أحمد(r • ץ): "تقويم نظم الإدارة البيئية للمؤسسات الحكومية " رسالة

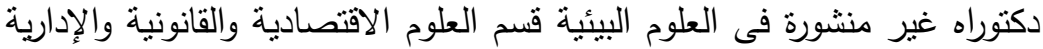

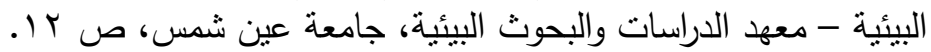

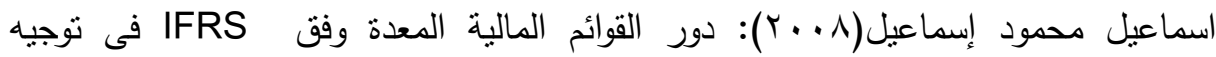
وتتجيع الاستثمارات، مجلة الفكر المحاسبى، كلية التجارة ،جامعة عين شمس،

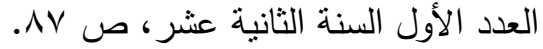

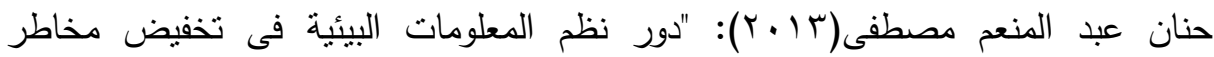

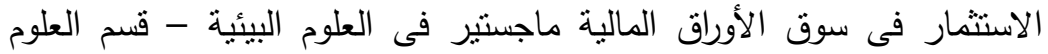

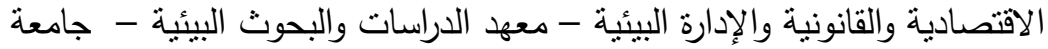

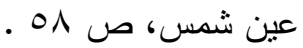

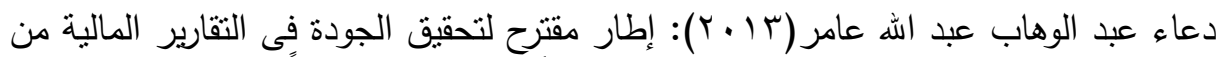

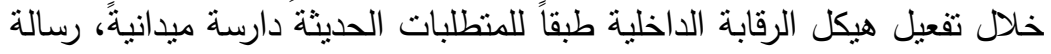

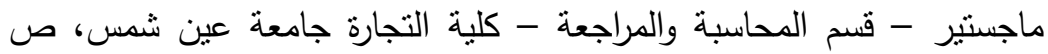

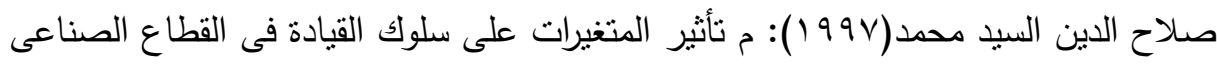

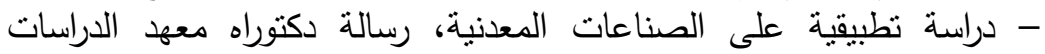

$$
\text { والبحوث البيئية - جامعة عين شمس ،صنم }
$$

عصام محمود مصطفى(ب(ץ): تفعيل نظم الإدارة البيئية فى المنشآت الصناعية بهدف

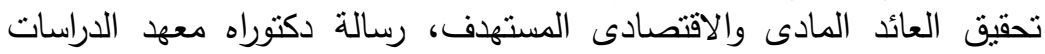

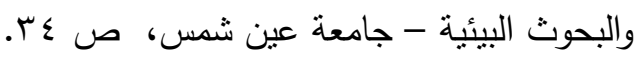

Bushman,R.M., J.D Piotroski, and A.J Smith : What Determines corporate Transparency? Journal Of Accounting Research.2004 issue 2,Vol.4 2p.234 
محمد عبد الفناح محمد وآخرون

Frederick H.wu (1994): Accounting information System thcorye Practice (Tokyo; Magraw Hill book Co.,)p,12

Jonas, GJ. \& J. Blanchet (2000): " Assessing Quality Of Financial Reporting "AAA American Accounting Association Accounting Horizons Vol. 14,No.3, September. pp353 363.

Lucas,H (1992) information systems concepts for management $\left(2^{\text {nd }}\right.$ ed) N.Y.MCGRAUL -Hill, P.8.

Tony Kang and Yang Hoong Pang (2005): "Economic Development and The Value Relevance of Accounting Information - A Disclosure Transparency Perspective " Review of Accounting \&Finance vol. 4,no.1.pp5 31. 


\title{
THE ROLE OF ENVIRONMENTAL INFORMATION SYSTEMS IN ENHANCING THE QUALITY OF FINANCIAL REPORTS AN APPLIED STUDY
}

Mohamed, M. A. ${ }^{(1)}$; Ramadan, Dalia, A. ${ }^{(1)}$ and Dorra, Inas. M. A.

1) Faculty of Commerce, Ain Shams University. 2) Institute of Environmental Studies and Research •Ain Shams University

\begin{abstract}
This study tackles with development of the accounting performance by providing and preparing financial statements in a way that fulfills the requirements of current stage, focusing on the role of environmental informatics and promotion of the accounting processing as well as processing data and information.

- Financial statements are critically important for their users at decision 'making of institutions.

- The commitment to transparency through taking specific procedures and rules leads to the increase of quality level concerning financial statements that could be achieved through environmental informatics.

The study concludes to the following:

* Proving validity of the main hypothesis that states a significant statistical relationship exists between efficacy of the environmental informatics and quality of financial statements.

* Proving validity of the second main hypothesis that shows a significant statistical impact between efficacy of the environmental information systems and quality of financial statements.
\end{abstract}

From those two major hypotheses, the following minor hypotheses are derived: 
محمد عبد الفتاح محمد وآخرون

- The First Hypothesis: There is a significant statistical impact of the effective system of environmental information on quality of financial statements.

- The Second Hypothesis: There is a significant statistical impact between requirements of quality in financial statements and quality in financial statements. 